\title{
Acute Anterior Myocardial Infarction by ECG Finding
}

National Cancer Institute

\section{Source}

National Cancer Institute. Acute Anterior Myocardial Infarction by ECG Finding. NCI

Thesaurus. Code C71065.

An electrocardiographic finding of patholog ic Q waves with accompanying ST elevation in leads V3 and V4, which is suggestive of acute myocardial infarction of the anterior wall of the left ventricle. (CDISC) 